Research Article

\title{
Permanence and Global Stability of Positive Periodic Solutions of a Discrete Competitive System
}

\author{
Wenjie Qin, Zhijun Liu, and Yiping Chen \\ Department of Mathematics, Hubei Institute for Nationalities, Enshi, Hubei 445000, China \\ Correspondence should be addressed to Zhijun Liu, zhijun_liu47@hotmail.com
}

Received 12 March 2009; Accepted 18 May 2009

Recommended by Antonia Vecchio

We consider the dynamic behaviors of a discrete competitive system. A good understanding of the permanence, existence, and global stability of positive periodic solutions is gained. Numerical simulations are also presented to substantiate the analytical results.

Copyright (C) 2009 Wenjie Qin et al. This is an open access article distributed under the Creative Commons Attribution License, which permits unrestricted use, distribution, and reproduction in any medium, provided the original work is properly cited.

\section{Introduction}

In biomathematics, one of the most challenging aspects of mathematical biology is competition modeling. Although the mathematical idea is simple [1], this type of modeling is so difficult to carry out in any generality since there are so many ways for a population to compete; many classical competitive models have been established to describe the relationships between species and the outer environment, and the connections between different species. Lotka-Volterra competitive model of two species communities is probably the best known model of mathematical ecology. In 1934, Gause [2] found out the competitive exclusion theory, which states that two species that compete for the exact same resources cannot stably coexist. One of the two competitors will always have an ever so slight advantage over the other that leads to extinction of the second competitor in the long run. Since then the competitive model has increasingly won attention as an important and fundament model in biomathematics. The dynamic relationship between species and their competitors has long been one of the dominant theses in both ecology and mathematical ecology. As a consequence, many excellent results concerned with permanence, extinction, stability and hopf bifurcations, and existence and global stability of positive periodic solutions of Lotka-Volterra competitive system are obtained (see [3-16]). 
Although much progress has been seen for Lotka-Volterra competitive systems, such systems are not well studied in the sense that most results are continuous time versions related. Many authors [17-21] have argued that the discrete-time models governed by difference equations are more appropriate than the continuous ones when populations have a short life expectancy, nonoverlapping generations in the real word. Discrete-time models can provide efficient computational models of continuous models for numerical simulations. So it is reasonable to study discrete-time competitive systems governed by difference equations.

In this paper, we will consider the dynamic behavior of a discrete-time competitive system. Let us first introduce its continuous time version which is motivated in [22]

$$
\begin{aligned}
& \dot{x}_{1}(t)=x_{1}(t)\left[r_{1}(t)-a_{1}(t) x_{1}(t)-\frac{c_{2}(t) x_{2}(t)}{1+x_{2}(t)}\right], \\
& \dot{x}_{2}(t)=x_{2}(t)\left[r_{2}(t)-a_{2}(t) x_{2}(t)-\frac{c_{1}(t) x_{1}(t)}{1+x_{1}(t)}\right],
\end{aligned}
$$

where $x_{1}(t), x_{2}(t)$ are the population densities of two competing species; $r_{1}(t), r_{2}(t)$ are the intrinsic growth rates of species; $a_{1}(t), a_{2}(t)$ are the rates of intraspecific competition of the first and second species, respectively; $c_{1}(t), c_{2}(t)$ are the rates of interspecific competition of the first and second species, respectively. All the coefficients above are continuous and bounded above and below by positive constants.

Following the same idea and method in [21], one can easily derive the discrete analogue of system (1.1), which takes the form of

$$
\begin{aligned}
& x_{1}(n+1)=x_{1}(n) \exp \left[r_{1}(n)-a_{1}(n) x_{1}(n)-\frac{c_{2}(n) x_{2}(n)}{1+x_{2}(n)}\right], \\
& x_{2}(n+1)=x_{2}(n) \exp \left[r_{2}(n)-a_{2}(n) x_{2}(n)-\frac{c_{1}(n) x_{1}(n)}{1+x_{1}(n)}\right],
\end{aligned}
$$

The exponential form of system (1.2) is more biologically reasonable than that directly derived by replacing the differential by difference in system (1.1) because this exponential form can assure $x_{i}(n+1)>0$ if $x_{i}(0)>0(i=1,2)$. Here $x_{i}(n)$ represent the densities of species $x_{i}$ at the $n$th generation, $r_{i}(n)$ are the intrinsic growth rates of species $x_{i}$ at the $n$th generation, $a_{i}(n)$ measure the intraspecific effects of the $n$th generation of species $x_{i}$ on own population, and $c_{i}(n)$ stand for the interspecific effects of the $n$th generation of species $x_{i}$ on species $x_{j}(i, j=1,2 ; i \neq j)$.

It is well known that, compared to the continuous time systems, the discrete-time ones are more difficult to deal with. The principle aim of this paper is to explore the permanence, existence, and global stability of positive periodic solutions of system (1.2). To the best of our knowledge, no work has been done for system (1.2).

For the sake of simplicity and convenience in the following discussion, the notations below will be used through this paper:

$$
f^{U}=\sup _{n \in \mathbb{N}} f(n), \quad f^{L}=\inf _{n \in \mathbb{N}} f(n),
$$

where $\{f(n)\}$ is a bounded sequence and $\mathbb{N}$ is the set of nonnegative integer numbers. 
For biological reasons, in system (1.2) we only consider the solution $\left\{x_{1}(n), x_{2}(n)\right\}$ with the initial value $\left\{x_{1}(0), x_{2}(0)\right\}>0$.

The organization of this paper is as follows. In the next section, we establish the permanence of system (1.2). In Section 3, we obtain sufficient conditions which ensure the existence and global stability of positive periodic solutions of system (1.2). Numerical simulations are present to illustrate the feasibility of our main results in final section.

\section{Permanence}

In this section, we will establish sufficient conditions for the permanence of system (1.2).

Definition 2.1. System (1.2) is said to be permanent if there exist positive constants $m_{i}$ and $M_{i}$ such that each positive solution $\left\{x_{1}(n), x_{2}(n)\right\}$ of system (1.2) satisfies

$$
m_{i} \leq \liminf _{n \rightarrow+\infty} x_{i}(n) \leq \limsup _{n \rightarrow+\infty} x_{i}(n) \leq M_{i}, \quad i=1,2
$$

Proposition 2.2. Any positive solution $\left\{x_{1}(n), x_{2}(n)\right\}$ of system (1.2) satisfies

$$
\limsup _{n \rightarrow+\infty} x_{i}(n) \leq M_{i} \stackrel{\text { def }}{=} \frac{\left[\exp \left(r_{i}^{U}-1\right)\right]}{a_{i}^{L}}, \quad i=1,2 .
$$

Proof. To prove Proposition 2.2, we consider Case 1 and Case 2.

Case 1. Assume that there exists an $n_{0} \in \mathbb{N}$ such that $x_{1}\left(n_{0}+1\right) \geq x_{1}\left(n_{0}\right)$, from the first equation of system (1.2), it follows that

$$
r_{1}\left(n_{0}\right)-a_{1}\left(n_{0}\right) x_{1}\left(n_{0}\right)-\frac{c_{2}\left(n_{0}\right) x_{2}\left(n_{0}\right)}{1+x_{2}\left(n_{0}\right)} \geq 0
$$

which implies,

$$
x_{1}\left(n_{0}\right) \leq \frac{r_{1}\left(n_{0}\right)}{a_{1}\left(n_{0}\right)} \leq \frac{r_{1}^{U}}{a_{1}^{L}} .
$$

Then

$$
\begin{aligned}
x_{1}\left(n_{0}+1\right) & =x_{1}\left(n_{0}\right) \exp \left[r_{1}\left(n_{0}\right)-a_{1}\left(n_{0}\right) x_{1}\left(n_{0}\right)-\frac{c_{2}\left(n_{0}\right) x_{2}\left(n_{0}\right)}{1+x_{2}\left(n_{0}\right)}\right] \\
& \leq x_{1}\left(n_{0}\right) \exp \left[r_{1}^{U}-a_{1}^{L} x_{1}\left(n_{0}\right)\right] \\
& \leq \frac{\left[\exp \left(r_{1}^{U}-1\right)\right]}{a_{1}^{L}} \\
& \stackrel{\text { def }}{=} M_{1},
\end{aligned}
$$


where we use the fact that $\max _{x \in \mathbb{R}}[x \exp (a-b x)]=[\exp (a-1)] / b$ for $a, b>0$, and $\mathbb{R}$ is the set of all real numbers. Hence $x_{1}\left(n_{0}\right) \leq M_{1}$.

We claim that $x_{1}(n) \leq M_{1}$ for all $n \geq n_{0}$. By way of contradiction, assume that there exists a $p_{0}>n_{0}$ such that $x_{1}\left(p_{0}\right)>M_{1}$, then $p_{0} \geq n_{0}+2$. Let

$$
\widehat{p}_{0}=\min \left\{p_{0}: p_{0} \geq n_{0}+2, x_{1}\left(p_{0}\right)>M_{1}\right\},
$$

that is, $x_{1}\left(\widehat{p}_{0}\right)>M_{1}$ and $\hat{p}_{0} \geq n_{0}+2$, then $x_{1}\left(\widehat{p}_{0}\right)>M_{1} \geq x_{1}\left(\hat{p}_{0}-1\right)$. It is easy to obtain that $x_{1}\left(\hat{p}_{0}\right) \leq M_{1}$ from the above argument, which is a contradiction. Therefore, $x_{1}(n) \leq M_{1}$ for all $n \geq n_{0}$, then $\lim \sup _{n \rightarrow+\infty} x_{1}(n) \leq M_{1}$. This proves the claim.

Case 2. Suppose that $x_{1}(n+1)<x_{1}(n)$ for all $n \in \mathbb{N}$. In particular, $\lim _{n \rightarrow+\infty} x_{1}(n)$ exists, denoted by $\bar{x}_{1}$, we will prove $\bar{x}_{1} \leq r_{1}^{U} / a_{1}^{L}$ by way of contradiction as follows. Assume that $\bar{x}_{1}>r_{1}^{U} / a_{1}^{L}$, taking limit in the first equation of system (1.2), which leads to

$$
\lim _{n \rightarrow+\infty}\left[r_{1}(n)-a_{1}(n) x_{1}(n)-\frac{c_{2}(n) x_{2}(n)}{1+x_{2}(n)}\right]=0,
$$

however,

$$
\begin{aligned}
\lim _{n \rightarrow+\infty}\left[r_{1}(n)-a_{1}(n) x_{1}(n)-\frac{c_{2}(n) x_{2}(n)}{1+x_{2}(n)}\right] & \leq \lim _{n \rightarrow+\infty}\left[r_{1}(n)-a_{1}(n) x_{1}(n)\right] \\
& \leq r_{1}^{U}-a_{1}^{L} \bar{x}_{1} \\
& <0
\end{aligned}
$$

which is a contradiction. This proves the claim. By the fact that $\min _{x \in \mathbb{R}^{+}}\{[\exp (x-1)] / x\}=1$, we obtain that $\bar{x}_{1} \leq r_{1}^{U} / a_{1}^{L} \leq r_{1}^{U} / a_{1}^{L} \cdot \exp \left(r_{1}^{U}-1\right) / r_{1}^{U} \stackrel{\text { def }}{=} M_{1}$. Therefore,

$$
\limsup _{n \rightarrow+\infty} x_{1}(n) \leq M_{1}=\frac{\left[\exp \left(r_{1}^{U}-1\right)\right]}{a_{1}^{L}}
$$

Analogously,

$$
\limsup _{n \rightarrow+\infty} x_{2}(n) \leq M_{2}=\frac{\left[\exp \left(r_{2}^{U}-1\right)\right]}{a_{2}^{L}} .
$$

This completes the proof of Proposition 2.2.

Proposition 2.3. Suppose that system (1.2) satisfies the following assumptions:

$$
r_{1}^{L}>c_{2}^{U}, \quad r_{2}^{L}>c_{1}^{U} .
$$


Then any positive solution $\left\{x_{1}(n), x_{2}(n)\right\}$ of system (1.2) satisfies

$$
\liminf _{n \rightarrow+\infty} x_{i}(n) \geq m_{i} \stackrel{\text { def }}{=} \frac{r_{i}^{L}-c_{j}^{U}}{a_{i}^{U}} \exp \left(r_{i}^{L}-a_{i}^{U} M_{i}-c_{j}^{U}\right), \quad i \neq j ; i, j=1,2 .
$$

Proof. By Proposition 2.2, since $\lim \sup _{n \rightarrow+\infty} x_{1}(n) \leq M_{1}$ for each $\varepsilon>0$, then there exists an $n^{*} \in \mathbb{N}$ such that $x_{1}(n) \leq M_{1}+\varepsilon$ for $n \geq n^{*}$. In order to prove Proposition 2.3, there are two cases to be considered as follows.

Case 1. Assume that there exists an $n_{0} \geq n^{*}$ such that $x_{1}\left(n_{0}+1\right) \leq x_{1}\left(n_{0}\right)$, by the first equation of system (1.2), it derives that

$$
\begin{aligned}
x_{1}\left(n_{0}+1\right) & =x_{1}\left(n_{0}\right) \exp \left[r_{1}\left(n_{0}\right)-a_{1}\left(n_{0}\right) x_{1}\left(n_{0}\right)-\frac{c_{2}\left(n_{0}\right) x_{2}\left(n_{0}\right)}{1+x_{2}\left(n_{0}\right)}\right] \\
& \geq x_{1}\left(n_{0}\right) \exp \left[r_{1}^{L}-a_{1}^{U} x\left(n_{0}\right)-c_{2}^{U}\right]
\end{aligned}
$$

therefore,

$$
r_{1}^{L}-a_{1}^{U} x\left(n_{0}\right)-c_{2}^{U} \leq 0
$$

It follows from the inequality (2.11) that

$$
x_{1}\left(n_{0}\right) \geq \frac{\left(r_{1}^{L}-c_{2}^{U}\right)}{a_{1}^{U}}>0
$$

By (2.13) and (2.15) we have

$$
\begin{aligned}
x_{1}\left(n_{0}+1\right) & =x_{1}\left(n_{0}\right) \exp \left[r_{1}\left(n_{0}\right)-a_{1}\left(n_{0}\right) x_{1}\left(n_{0}\right)-\frac{c_{2}\left(n_{0}\right) x_{2}\left(n_{0}\right)}{1+x_{2}\left(n_{0}\right)}\right] \\
& \geq \frac{r_{1}^{L}-c_{2}^{U}}{a_{1}^{U}} \exp \left[r_{1}^{L}-a_{1}^{U}\left(M_{1}+\varepsilon\right)-c_{2}^{U}\right] \\
& >0 .
\end{aligned}
$$

Hence $x_{1}\left(n_{0}\right) \geq x_{1}^{\varepsilon}$, where $x_{1}^{\varepsilon} \stackrel{\text { def }}{=}\left(\left(r_{1}^{L}-c_{2}^{U}\right) / a_{1}^{U}\right) \exp \left[r_{1}^{L}-a_{1}^{U}\left(M_{1}+\varepsilon\right)-c_{2}^{U}\right]$.

In the following we will prove $x_{1}(n) \geq x_{1}^{\varepsilon}$ for all $n \geq n_{0}$. By way of contradiction, assume that there exists a $p_{0}>n_{0}$ such that $x_{1}\left(p_{0}\right)<x_{1}^{\varepsilon}$, then $p_{0} \geq n_{0}+2$. Let

$$
\tilde{p}_{0}=\min \left\{p_{0}: p_{0} \geq n_{0}+2, x_{1}\left(p_{0}\right)<x_{1}^{\varepsilon}\right\},
$$


that is, $x_{1}\left(\tilde{p}_{0}\right)<x_{1}^{\varepsilon}$ and $\tilde{p}_{0} \geq n_{0}+2$, then $x_{1}\left(\tilde{p}_{0}\right)<x_{1}^{\varepsilon} \leq x_{1}\left(\tilde{p}_{0}-1\right)$, the above argument produces that $x_{1}\left(\tilde{p}_{0}\right) \geq x_{1}^{\varepsilon}$, which is a contradiction. Therefore, $x_{1}(n) \geq x_{1}^{\varepsilon}$ for all $n \geq n_{0}$, since $\varepsilon$ can be sufficiently small, it gives that

$$
x_{1}(n) \geq \frac{r_{1}^{L}-c_{2}^{U}}{a_{1}^{U}} \exp \left(r_{1}^{L}-a_{1}^{U} M_{1}-c_{2}^{U}\right) \stackrel{\text { def }}{=} m_{1}>0,
$$

then $\lim \inf _{n \rightarrow+\infty} x_{1}(n) \geq m_{1}$. This proves the claim.

Case 2. Assume that $x_{1}(n+1)>x_{1}(n)$ for a sufficiently large $n \geq n^{*}$. In this case, $\lim _{n \rightarrow+\infty} x_{1}(n)$ exists, denoted by $\underline{x}_{1}$. For the sake of proving $x_{1} \geq\left(r_{1}^{L}-c_{2}^{U}\right) / a_{1}^{U}$, by way of contradiction, assume that $\underline{x}_{1}<\left(r_{1}^{L}-c_{2}^{U}\right) / a_{1}^{U}$, taking limit in the first equation of system (1.2), it follows that

$$
\lim _{n \rightarrow+\infty}\left[r_{1}(n)-a_{1}(n) x_{1}(n)-\frac{c_{2}(n) x_{2}(n)}{1+x_{2}(n)}\right]=0,
$$

however,

$$
\begin{aligned}
\lim _{n \rightarrow+\infty}\left[r_{1}(n)-a_{1}(n) x_{1}(n)-\frac{c_{2}(n) x_{2}(n)}{1+x_{2}(n)}\right] & \geq \lim _{n \rightarrow+\infty}\left[r_{1}(n)-a_{1}(n) x_{1}(n)-c_{2}(n)\right] \\
& \geq r_{1}^{L}-a_{1}^{U} \underline{x}_{1}-c_{2}^{U} \\
& >0
\end{aligned}
$$

which is a contradiction. It implies that $\underline{x}_{1} \geq\left(r_{1}^{L}-c_{2}^{U}\right) / a_{1}^{U}$. By the fact that $\min _{x \in \mathbb{R}^{+}}\{[\exp (x-$ $1)] / x\}=1$, we obtain that $M_{1}=\left[\exp \left(r_{1}^{U}-1\right)\right] / a_{1}^{L} \geq r_{1}^{U} / a_{1}^{L} \geq r_{1}^{L} / a_{1}^{U}$. Thus $\underline{x}_{1}>\left(r_{1}^{L}-c_{1}^{U}\right) / a_{1}^{U} \geq$ $x_{1}^{\varepsilon}$. Therefore, $\lim \inf _{n \rightarrow+\infty} x_{1}(n) \geq x_{1}^{\varepsilon}$. Since $\varepsilon$ can be sufficiently small, we have

$$
\liminf _{n \rightarrow+\infty} x_{1}(n) \geq m_{1} \stackrel{\text { def }}{=} \frac{r_{1}^{L}-c_{2}^{U}}{a_{1}^{U}} \exp \left(r_{1}^{L}-a_{1}^{U} M_{1}-c_{2}^{U}\right)>0 .
$$

Analogously, by the second inequality in (2.11), we can obtain that

$$
\liminf _{n \rightarrow+\infty} x_{2}(n) \geq m_{2} \stackrel{\text { def }}{=} \frac{r_{2}^{L}-c_{1}^{U}}{a_{2}^{U}} \exp \left(r_{2}^{L}-a_{2}^{U} M_{2}-c_{1}^{U}\right)>0
$$

This completes the proof of Proposition 2.3.

Now, we are in a position to state Theorem 2.4 whose proof is a direct consequence of Propositions 2.2 and 2.3 .

Theorem 2.4. If the inequalities in (2.11) hold, then system (1.2) is permanent. 


\section{Existence and Global Stability of Positive Periodic Solutions}

In this section, we suppose system (1.2) is a periodic system, and then we investigate the existence and global stability of positive periodic solutions of such system. To do this, assume that all the coefficients of system (1.2) are $\omega$-periodic, in other words,

$$
r_{i}(n+\omega)=r_{i}(n), \quad a_{i}(n+\omega)=a_{i}(n), \quad c_{i}(n+\omega)=c_{i}(n), \quad i=1,2
$$

Theorem 3.1. If the inequalities in (2.11) hold, then system (1.2) has at least one strictly positive $\omega$-periodic solution, denoted by $\left\{x_{1}^{*}(n), x_{2}^{*}(n)\right\}$.

Proof. We know that $K=\left[m_{1}, M_{1}\right] \times\left[m_{2}, M_{2}\right]$ is an invariant set of system (1.2) from Propositions 2.2 and 2.3. Define the continuous mapping $F$ on $K$

$$
F\left\{x_{1}^{*}(0), x_{2}^{*}(0)\right\}=\left\{x_{1}^{*}(\omega), x_{2}^{*}(\omega)\right\}, \quad \text { for }\left\{x_{1}^{*}(0), x_{2}^{*}(0)\right\} \in K
$$

Obviously, $F$ depends continuously on $\left\{x_{1}^{*}(0), x_{2}^{*}(0)\right\}$, then $F$ is continuous and maps the compact set $K=\left[m_{1}, M_{1}\right] \times\left[m_{2}, M_{2}\right]$ into itself. Therefore, $F$ has a fixed point $\left\{x_{1}^{*}, x_{2}^{*}\right\}$. It is easy to see that the solution $\left\{x_{1}^{*}(n), x_{2}^{*}(n)\right\}$ which passes through $\left\{x_{1}^{*}, x_{2}^{*}\right\}$ is an $\omega$-periodic solution of system (1.2). The proof is complete.

Next, we derive sufficient conditions which guarantee that the positive periodic solution of system (1.2) is globally stable. We first give the definition of global stability.

Definition 3.2. A positive periodic solution $\left\{x_{1}^{*}(n), x_{2}^{*}(n)\right\}$ of system (1.2) is globally stable if each other solution $\left\{x_{1}(n), x_{2}(n)\right\}$ of system (1.2) with positive initial value defined for all $n>0$ satisfies

$$
\lim _{n \rightarrow+\infty}\left|x_{1}(n)-x_{1}^{*}(n)\right|=0, \quad \lim _{n \rightarrow+\infty}\left|x_{2}(n)-x_{2}^{*}(n)\right|=0 .
$$

Now, we give the main result in this section.

Theorem 3.3. In addition to (2.11), assume further that the following assumptions

$$
\begin{aligned}
& \lambda_{1} \stackrel{\text { def }}{=} \max \left\{\left|1-a_{1}^{L} m_{1}\right|,\left|1-a_{1}^{U} M_{1}\right|\right\}+c_{2}^{U}<1, \\
& \lambda_{2} \stackrel{\text { def }}{=} \max \left\{\left|1-a_{2}^{L} m_{2}\right|,\left|1-a_{2}^{U} M_{2}\right|\right\}+c_{1}^{U}<1
\end{aligned}
$$

hold. Then the positive periodic solution of system (1.2) is globally stable. 


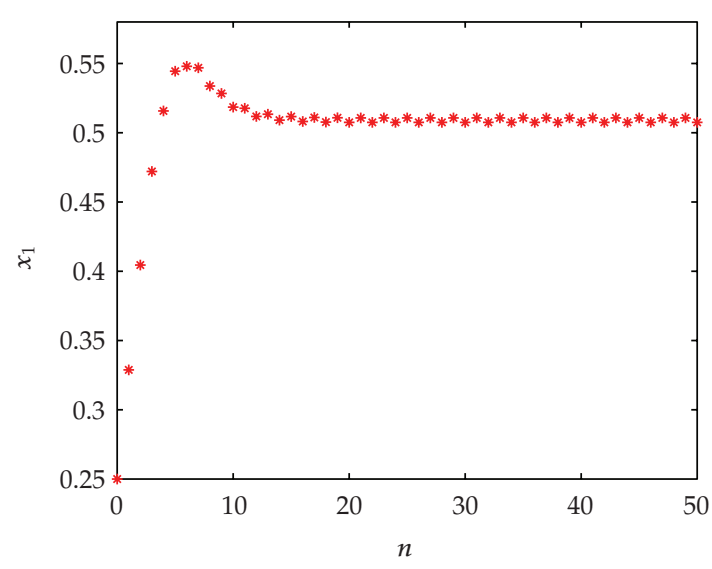

(a)

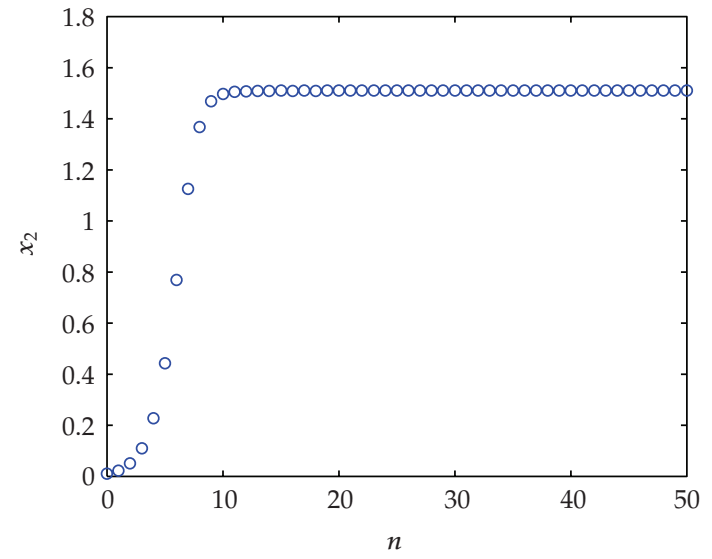

(b)

Figure 1: Permanence of system (1.2) with initial value $(0.25,0.01)$. (a) Time series of $x_{1}$ for $n \in[0,50]$. (b) Time series of $x_{2}$ for $n \in[0,50]$.

Proof. Let $\left\{x_{1}^{*}(n), x_{2}^{*}(n)\right\}$ be a positive periodic solution of system (1.2). We make the change of variables $x_{1}(n)=x_{1}^{*}(n) \exp u(n)$ and $x_{2}(n)=x_{2}^{*}(n) \exp v(n)$, then system (1.2) is rewritten as

$$
\begin{aligned}
& u(n+1)-u(n)=a_{1}(n) x_{1}^{*}(n)[1-\exp u(n)]+c_{2}(n)\left\{\frac{1}{1+x_{2}^{*}(n) \exp [v(n)]}-\frac{1}{1+x_{2}^{*}(n)}\right\} \\
& v(n+1)-v(n)=a_{2}(n) x_{2}^{*}(n)[1-\exp v(n)]+c_{1}(n)\left\{\frac{1}{1+x_{1}^{*}(n) \exp [u(n)]}-\frac{1}{1+x_{1}^{*}(n)}\right\}
\end{aligned}
$$

By the mean-value theorem, it derives that

$$
\begin{aligned}
& u(n+1)=u(n)\left\{1-a_{1}(n) x_{1}^{*}(n) \exp \left[\theta_{1} u(n)\right]\right\}-c_{2}(n) v(n) \frac{x_{2}^{*}(n) \exp \left[\theta_{2} v(n)\right]}{\left\{1+x_{2}^{*}(n) \exp \left[\theta_{2} v(n)\right]\right\}^{2}}, \\
& v(n+1)=v(n)\left\{1-a_{2}(n) x_{2}^{*}(n) \exp \left[\theta_{3} v(n)\right]\right\}-c_{1}(n) u(n) \frac{x_{1}^{*}(n) \exp \left[\theta_{4} u(n)\right]}{\left\{1+x_{1}^{*}(n) \exp \left[\theta_{4} u(n)\right]\right\}^{2}},
\end{aligned}
$$

where the constants $\theta_{1}, \theta_{2}, \theta_{3}, \theta_{4} \in(0,1)$.

Now, by (3.4) and (3.5), we choose the constant $\varepsilon$ sufficiently small such that

$$
\begin{aligned}
& \lambda_{1}^{\varepsilon}=\max \left\{\left|1-a_{1}^{L}\left(m_{1}-\varepsilon\right)\right|,\left|1-a_{1}^{U}\left(M_{1}+\varepsilon\right)\right|\right\}+c_{2}^{U}<1, \\
& \lambda_{2}^{\varepsilon}=\max \left\{\left|1-a_{2}^{L}\left(m_{2}-\varepsilon\right)\right|,\left|1-a_{2}^{U}\left(M_{2}+\varepsilon\right)\right|\right\}+c_{1}^{U}<1 .
\end{aligned}
$$


In view of Propositions 2.2 and 2.3, there exists an $n_{0} \in N$ such that $n \geq n_{0}$, we have

$$
\begin{array}{ll}
0<m_{1}-\varepsilon \leq x_{1}^{*}(n) \leq M_{1}+\varepsilon, & 0<m_{1}-\varepsilon \leq x_{1}(n) \leq M_{1}+\varepsilon, \\
0<m_{2}-\varepsilon \leq x_{2}^{*}(n) \leq M_{2}+\varepsilon, & 0<m_{2}-\varepsilon \leq x_{2}(n) \leq M_{2}+\varepsilon
\end{array}
$$

Since $\theta_{1}, \theta_{2}, \theta_{3}, \theta_{4} \in(0,1)$, both $x_{1}^{*}(n) \exp \left[\theta_{1} u(n)\right]$ and $x_{1}^{*}(n) \exp \left[\theta_{4} u(n)\right]$ are between $x_{1}(n)$ and $x_{1}^{*}(n)$. Meanwhile, both $x_{2}^{*}(n) \exp \left[\theta_{2} v(n)\right]$ and $x_{2}^{*}(n) \exp \left[\theta_{3} v(n)\right]$ are between $x_{2}(n)$ and $x_{2}^{*}(n)$. From the first equation of system (3.7), it follows that

$$
\begin{aligned}
|u(n+1)| & \leq|u(n)|\left|1-a_{1}(n) x_{1}^{*}(n) \exp \left[\theta_{1} u(n)\right]\right|+\left|c_{2}(n) v(n) \frac{x_{2}^{*}(n) \exp \left[\theta_{2} v(n)\right]}{1+x_{2}^{*}(n) \exp \left[\theta_{2} v(n)\right]}\right| \\
& \leq \max \left\{\left|1-a_{1}^{L}\left(m_{1}-\varepsilon\right)\right|,\left|1-a_{1}^{U}\left(M_{1}+\varepsilon\right)\right|\right\}|u(n)|+\max \left\{\left|c_{2}(n) v(n)\right|\right\} \\
& \leq \max \left\{\left|1-a_{1}^{L}\left(m_{1}-\varepsilon\right)\right|,\left|1-a_{1}^{U}\left(M_{1}+\varepsilon\right)\right|\right\}|u(n)|+c_{2}^{U}|v(n)| \\
& \leq \lambda_{1}^{\varepsilon} \max \{|u(n)|,|v(n)|\} .
\end{aligned}
$$

Similarly, it follows from (3.5) that

$$
|v(n+1)| \leq \lambda_{2}^{\varepsilon} \max \{|u(n)|,|v(n)|\}
$$

Denote $\lambda^{\varepsilon}=\max \left\{\lambda_{1}^{\varepsilon}, \lambda_{2}^{\varepsilon}\right\}$, then $\lambda^{\varepsilon}<1$. Therefore, when $n \geq n_{0}$,

$$
\max \{|u(n+1)|,|v(n+1)|\} \leq \lambda^{\varepsilon} \max \{|u(n)|,|v(n)|\} \leq\left(\lambda^{\varepsilon}\right)^{n-n_{0}} \max \left\{\left|u\left(n_{0}\right)\right|,\left|v\left(n_{0}\right)\right|\right\},
$$

as a consequence, $\lim _{n \rightarrow+\infty}\left|x_{1}(n)-x_{1}^{*}(n)\right|=0, \lim _{n \rightarrow+\infty}\left|x_{2}(n)-x_{2}^{*}(n)\right|=0$. By using Definition 3.2, it follows that the positive periodic solution $\left\{x_{1}^{*}(n), x_{2}^{*}(n)\right\}$ of system (1.2) is globally stable. This completes the proof.

Remark 3.4. Theorem 3.3 shows that $\left\{x_{1}^{*}(n), x_{2}^{*}(n)\right\}$ is the global attractor of all positive solutions of system (1.2), then $\left\{x_{1}^{*}(n), x_{2}^{*}(n)\right\}$ is the unique positive periodic solution of system (1.2).

\section{Example and Numerical Simulation}

In this paper, we have investigated the permanence and global stability of positive periodic solutions of a discrete competitive system. Each species is not isolated from its living environment, but competes with the other for the same resource. Sufficient conditions which guarantee the permanence, existence and global stability of positive periodic solutions are established, respectively. The theoretical results are confirmed by the following examples and their numerical results. 


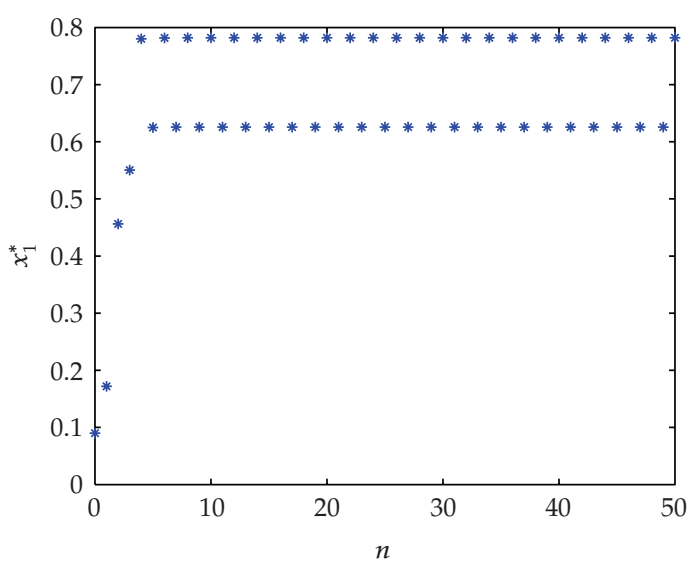

* $x_{1}^{*}(0)=0.09$

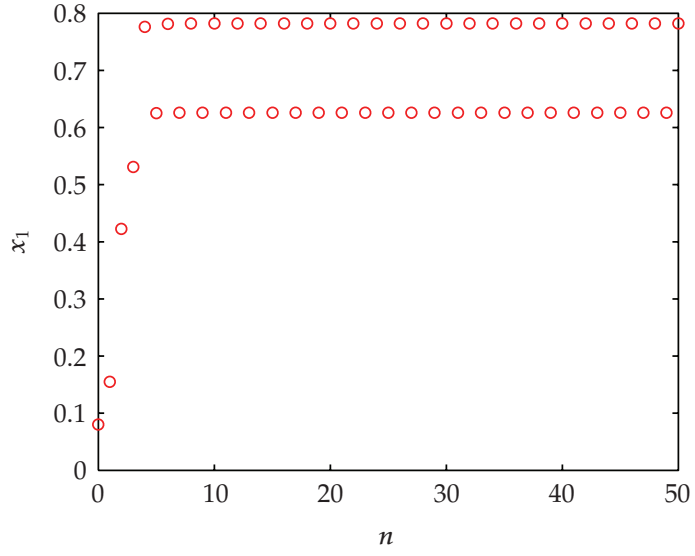

$\circ x_{1}(0)=0.08$

(a)

(b)

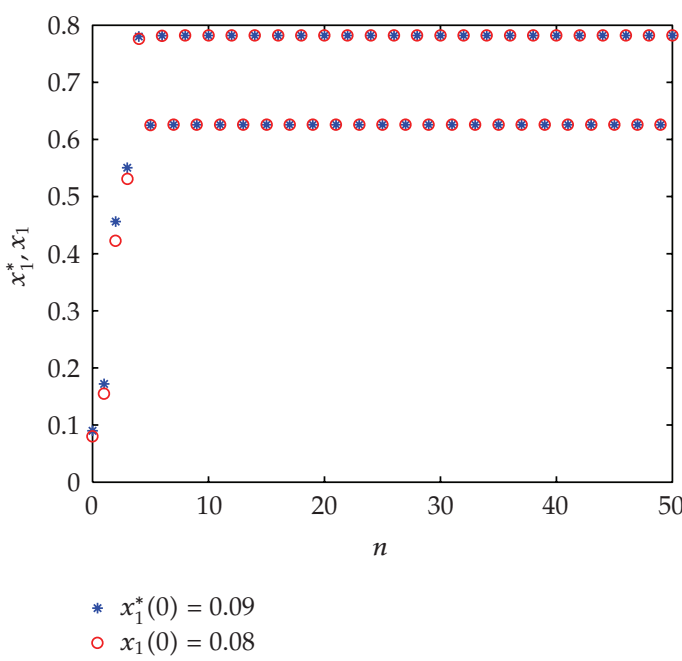

(c)

Figure 2: (a) Time series of $x_{1}^{*}$ with $x_{1}^{*}(0)=0.09$ for $n \in[0,50]$. (b) Time series of $x_{1}$ with $x_{1}(0)=0.08$ for $n \in[0,50]$. (c) Time series of $x_{1}^{*}$ and $x_{1}$ for $n \in[0,50]$ in the same coordinate system.

To verify the sufficient conditions for permanence of system (1.2), we assume that $r_{1}(n)=0.46+0.01 \sin \pi n, r_{2}(n)=0.88-0.02 \sin \pi n, a_{1}(n)=0.75+0.01 \cos \pi n, a_{2}(n)=0.52+$ $0.01 \sin \pi n, c_{1}(n)=0.28+0.02 \sin \pi n, c_{2}(n)=0.13-0.01 \sin \pi n$, and the initial condition $\left(x_{1}(0), x_{2}(0)\right)=(0.25,0.01)$. Clearly, $(2.11)$ in Theorem 2.4 are satisfied, and hence system (1.2) is permanent (see Figure 1).

Now, we further verify the sufficient conditions for the existence and global stability of positive periodic solutions of periodic system (1.2). Let us assume that all the coefficients of system (1.2) are periodic and listed in Table 1.

Besides, we choose the positive periodic solution with initial values $(0.09,0.01)$, denoted by $\left(x_{1}^{*}, x_{2}^{*}\right)$, and the positive solution with initial value $(0.08,0.02)$ denoted by $\left(x_{1}, x_{2}\right)$. By Theorem 3.3, a simple calculation shows that the assumptions in (3.4) and (3.5) hold. 


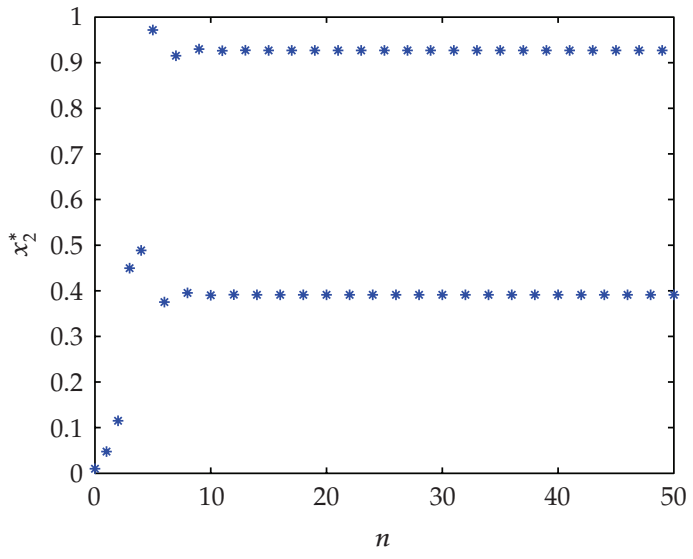

* $x_{2}^{*}(0)=0.01$

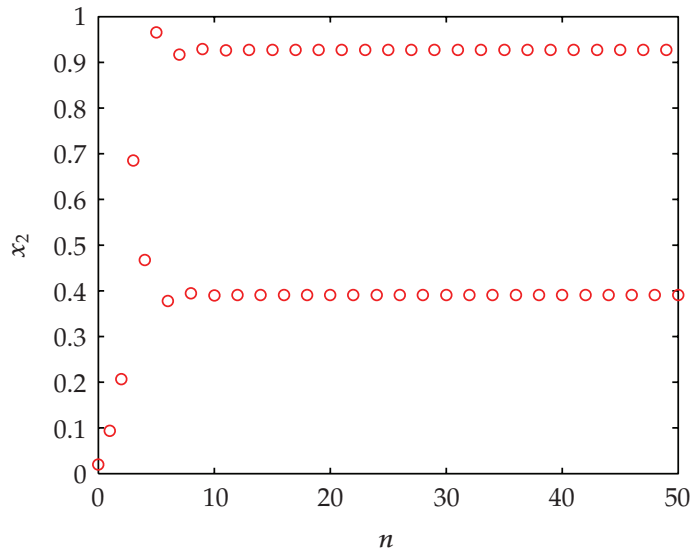

○ $x_{2}(0)=0.02$

(a)

(b)

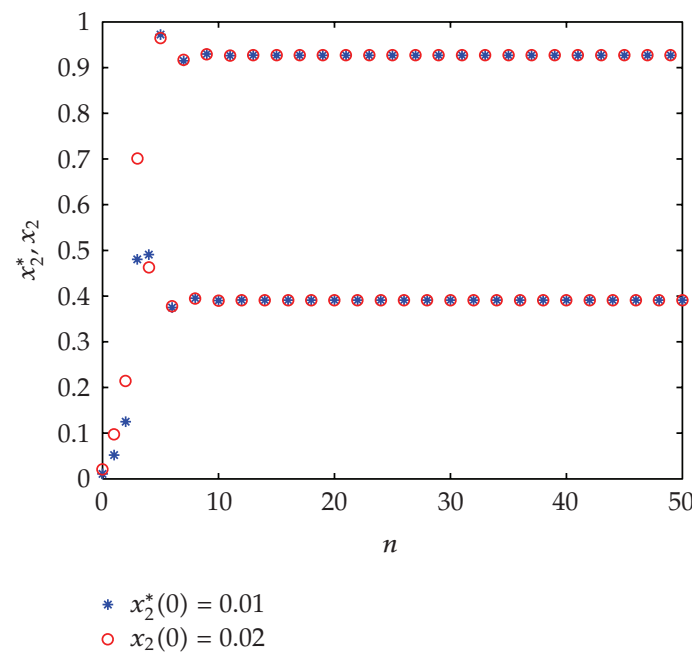

(c)

Figure 3: (a) Time series of $x_{2}^{*}$ with $x_{2}^{*}(0)=0.01$ for $n \in[0,50]$. (b) Time series of $x_{2}$ with $x_{2}(0)=0.02$ for $n \in[0,50]$. (c) Time series of $x_{2}^{*}$ and $x_{2}$ for $n \in[0,50]$ in the same coordinate system.

Table 1: The coefficient values when $\mathbf{n}$ is under different conditions.

\begin{tabular}{lcccccc}
\hline$n$ & $r_{1}(n)$ & $r_{2}(n)$ & $a_{1}(n)$ & $a_{2}(n)$ & $c_{1}(n)$ & $c_{2}(n)$ \\
\hline Odd number & 0.76 & 1.58 & 1.25 & 1.80 & 0.03 & 0.02 \\
Even number & 1.26 & 0.98 & 1.65 & 1.98 & 0.02 & 0.01 \\
\hline
\end{tabular}

So from Theorem 3.3 we know that periodic system (1.2) has a positive 2-periodic solution which is globally stable. From Figures 2(a), 2(b), and 2(c), we see that $x_{1}$ with $x_{1}(0)=0.08$ will tend to $x_{1}^{*}$ with $x_{1}^{*}(0)=0.09$. Similarly, from Figures $3(\mathrm{a}), 3(\mathrm{~b})$, and 3(c), we see that $x_{2}$ with $x_{2}(0)=0.02$ will tend to $x_{2}^{*}$ with $x_{2}^{*}(0)=0.01$. Furthermore, Figures $4(\mathrm{a})$ and $4(\mathrm{~b})$ show the phase portrait of periodic system $(1.2)$ with $\left(x_{1}^{*}(0), x_{2}^{*}(0)\right)=(0.09,0.01)$ for $n \in[0,50]$ 


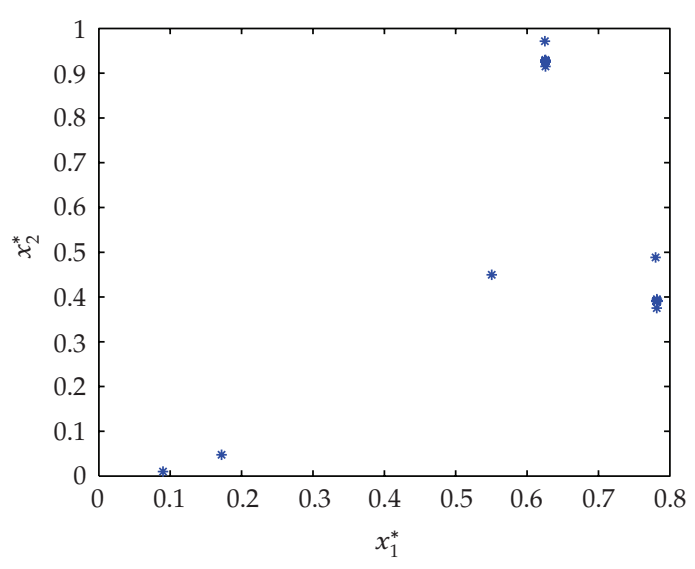

* $\left(x_{1}^{*}(0), x_{2}^{*}(0)\right)=(0.09,0.01)$

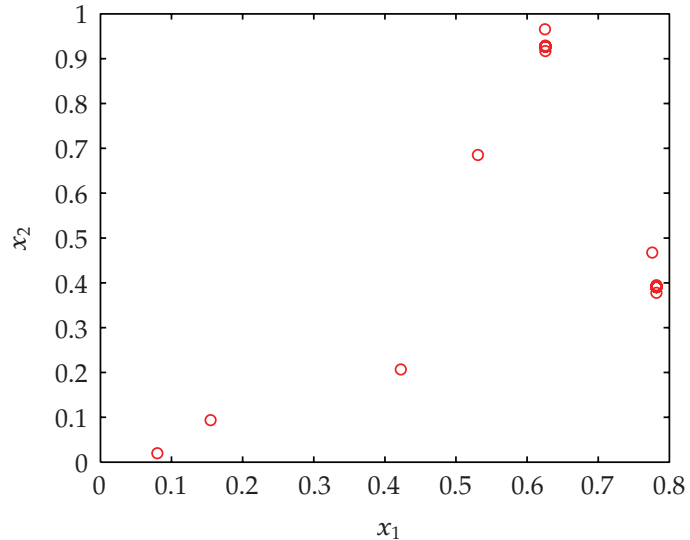

○ $\left(x_{1}(0), x_{2}(0)\right)=(0.08,0.02)$

(a)

(b)

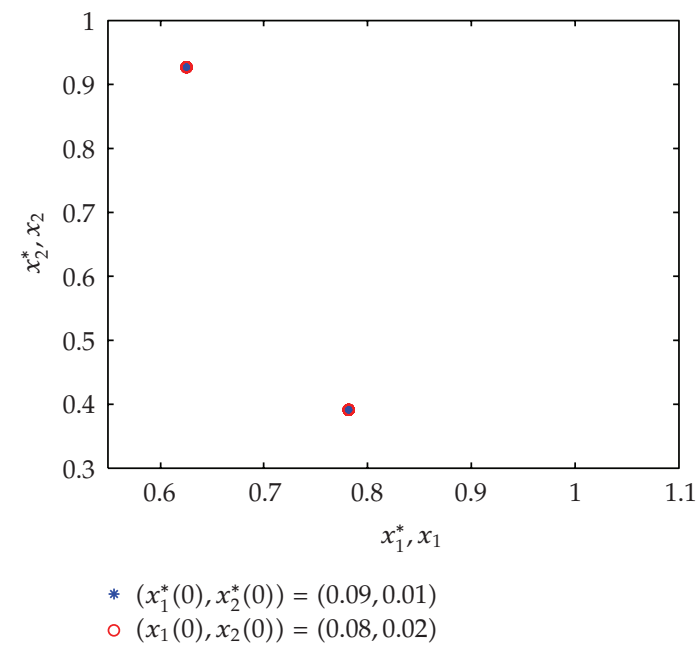

(c)

Figure 4: (a) Phase portrait of $x_{1}^{*}$ and $x_{2}^{*}$ with initial value $(0.09,0.01)$ for $n \in[0,50]$. (b) Phase portrait of $x_{1}$ and $x_{2}$ with initial value $(0.08,0.02)$ for $n \in[0,50]$. (c) Phase portraits of $x_{1}^{*}, x_{2}^{*}$ and $x_{1}, x_{2}$ for $n \in[30,50]$ in the same coordinate system.

and $\left(x_{1}(0), x_{2}(0)\right)=(0.08,0.02)$ for $n \in[0,50]$, respectively. From Figure $4(\mathrm{c})$, we can see that periodic system (1.2) has a positive 2-periodic solution which is globally stable.

\section{Acknowledgement}

The work is supported by the Innovation Term of Educational Department of Hubei Province in China (T200804), the National Science Foundation of Hubei Province in China (2008CDB068), and the Innovation Project of Hubei Institute for Nationalities for postgraduate students. We would like to thank the Editor Professor A. Vecchio and the referee for careful reading of the original manuscript and valuable comments and suggestions that greatly improved the presentation of this work. 


\section{References}

[1] H. L. Smith and P. Waltman, The Theory of the Chemostat: Dynamics of Microbial Competition, vol. 13 of Cambridge Studies in Mathematical Biology, Cambridge University Press, Cambridge, UK, 1995.

[2] G. F. Gause, The Struggle for Existence, Williams and Wilkins, Baltimare, Md, USA, 1934.

[3] B. Liu and L. Chen, "The periodic competing Lotka-Volterra model with impulsive effect," Mathematical Medicine and Biology, vol. 21, no. 2, pp. 129-145, 2004.

[4] S. Ahmad, "On the nonautonomous Volterra-Lotka competition equations," Proceedings of the American Mathematical Society, vol. 117, no. 1, pp. 199-204, 1993.

[5] S. Ahmad and F. Montes de Oca, "Average growth and extinction in a two dimensional Lotka-Volterra system," Dynamics of Continuous, Discrete E Impulsive Systems. Series A, vol. 9, no. 2, pp. 177-186, 2002.

[6] J. C. Eilbeck and J. López-Gómez, "On the periodic Lotka-Volterra competition model," Journal of Mathematical Analysis and Applications, vol. 210, no. 1, pp. 58-87, 1997.

[7] B. Lisena, "Competitive exclusion in a periodic Lotka-Volterra system," Applied Mathematics and Computation, vol. 177, no. 2, pp. 761-768, 2006.

[8] F. Montes de Oca and M. Vivas, "Extinction in two dimensional Lotka-Volterra system with infinite delay," Nonlinear Analysis: Real World Applications, vol. 7, no. 5, pp. 1042-1047, 2006.

[9] A. Tineo, "Necessary and sufficient conditions for extinction of one species," Advanced Nonlinear Studies, vol. 5, no. 1, pp. 57-71, 2005.

[10] Y. Song, M. Han, and Y. Peng, "Stability and Hopf bifurcations in a competitive Lotka-Volterra system with two delays," Chaos, Solitons \& Fractals, vol. 22, no. 5, pp. 1139-1148, 2004.

[11] X. H. Tang and X. Zou, "Global attractivity of non-autonomous Lotka-Volterra competition system without instantaneous negative feedback," Journal of Differential Equations, vol. 192, no. 2, pp. 502$535,2003$.

[12] Z. Y. Lu and Y. Takeuchi, "Permanence and global attractivity for competitive Lotka-Volterra systems with delay," Nonlinear Analysis: Theory, Methods \& Applications, vol. 22, no. 7, pp. 847-856, 1994.

[13] Z. Teng, "On the non-autonomous Lotka-Volterra $n$-species competing systems," Applied Mathematics and Computation, vol. 114, no. 2-3, pp. 175-185, 2000.

[14] Y. Li and Y. Kuang, "Periodic solutions of periodic delay Lotka-Volterra equations and systems," Journal of Mathematical Analysis and Applications, vol. 255, no. 1, pp. 260-280, 2001.

[15] Z. Liu, R. Tan, and Y. Chen, "Modeling and analysis of a delayed competitive system with impulsive perturbations," The Rocky Mountain Journal of Mathematics, vol. 38, no. 5, pp. 1505-1523, 2008.

[16] M. Fan, K. Wang, and D. Jiang, "Existence and global attractivity of positive periodic solutions of periodic $n$-species Lotka-Volterra competition systems with several deviating arguments," Mathematical Biosciences, vol. 160, no. 1, pp. 47-61, 1999.

[17] R. M. May, Stability and Complexity in Model Ecosystems, Princeton Unversity Press, Princeton, NJ, USA, 1974.

[18] H. I. Freedman, Deterministic Mathematical Models in Population Ecology, vol. 57 of Monographs and Textbooks in Pure and Applied Mathematics, Marcel Dekker, New York, NY, USA, 1980.

[19] R. P. Agarwal, Difference Equations and Inequalities: Theory, Methods, and Applications, vol. 228 of Monographs and Textbooks in Pure and Applied Mathematics, Marcel Dekker, New York, NY, USA, 2nd edition, 2000.

[20] J. D. Murray, Mathematical Biology, vol. 19 of Biomathematics, Springer, Berlin, Germany, 1989.

[21] M. Fan and K. Wang, "Periodic solutions of a discrete time nonautonomous ratio-dependent predatorprey system," Mathematical and Computer Modelling, vol. 35, no. 9-10, pp. 951-961, 2002.

[22] K. Gopalsamy, Stability and Oscillations in Delay Differential Equations of Population Dynamics, vol. 74 of Mathematics and Its Applications, Kluwer Academic Publishers, Dordrecht, The Netherlands, 1992. 


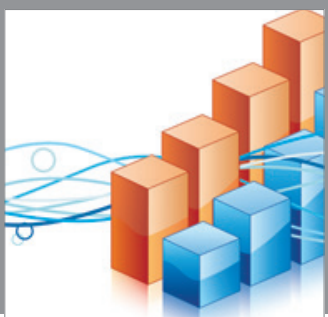

Advances in

Operations Research

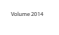

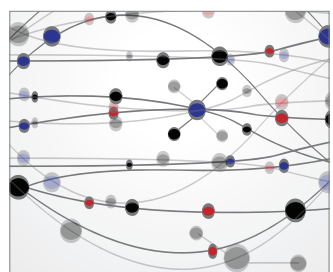

\section{The Scientific} World Journal
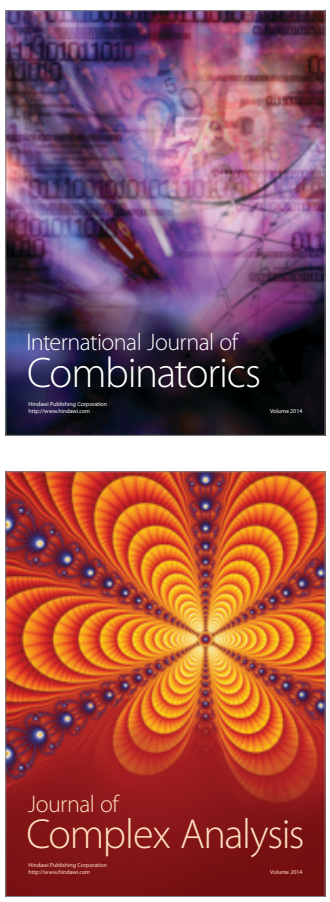

International Journal of

Mathematics and

Mathematical

Sciences
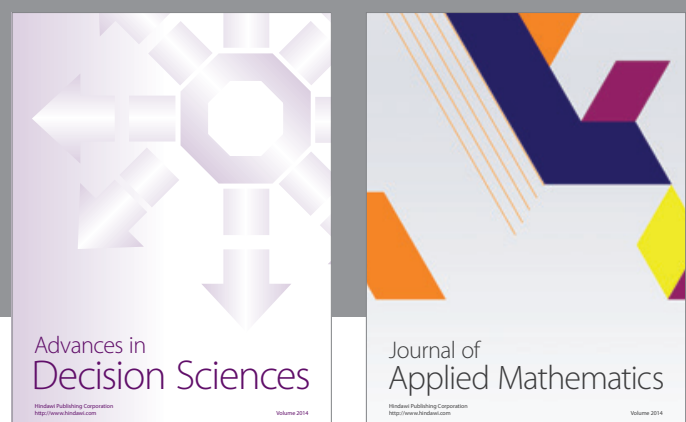

Journal of

Applied Mathematics
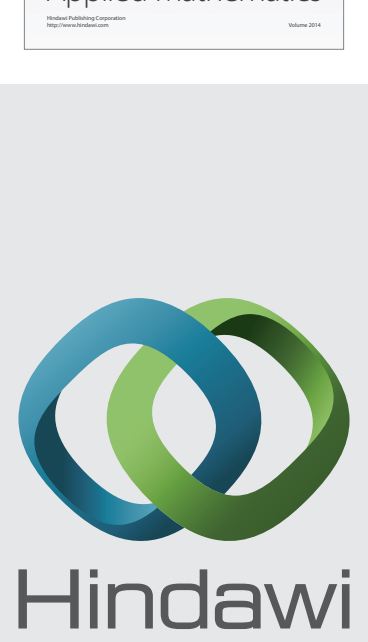

Submit your manuscripts at http://www.hindawi.com
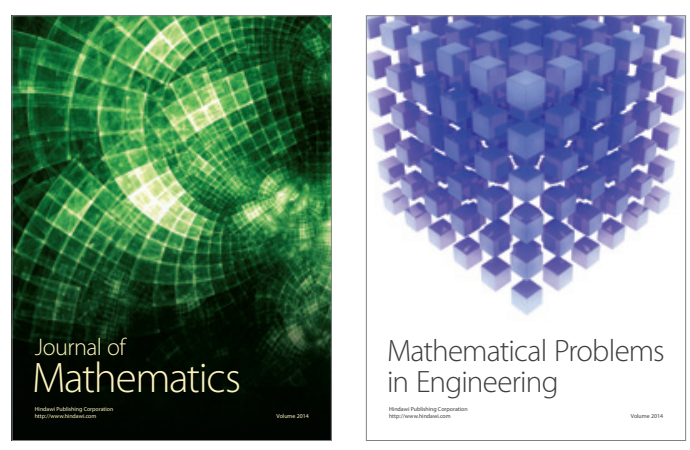

Mathematical Problems in Engineering
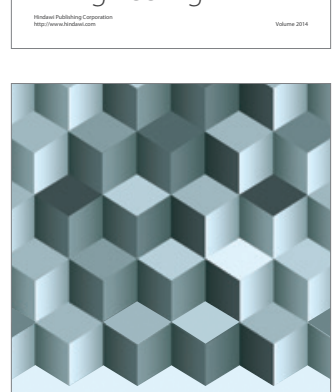

Journal of

Function Spaces
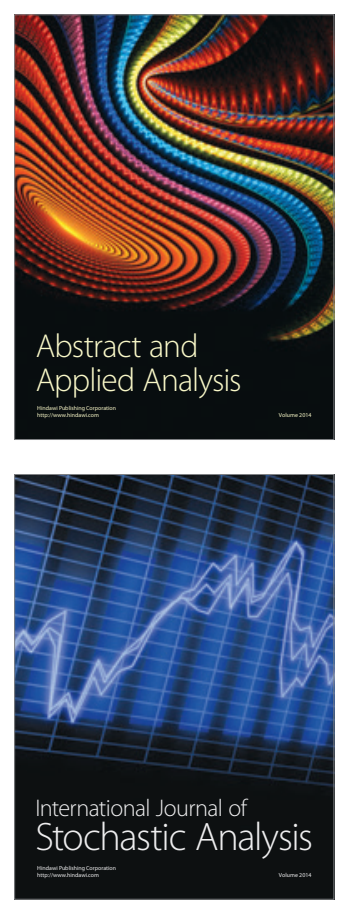

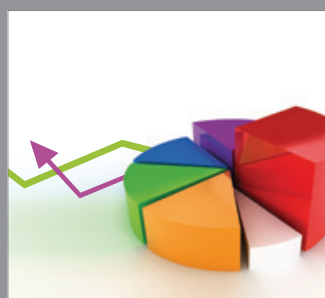

ournal of

Probability and Statistics

Promensencen
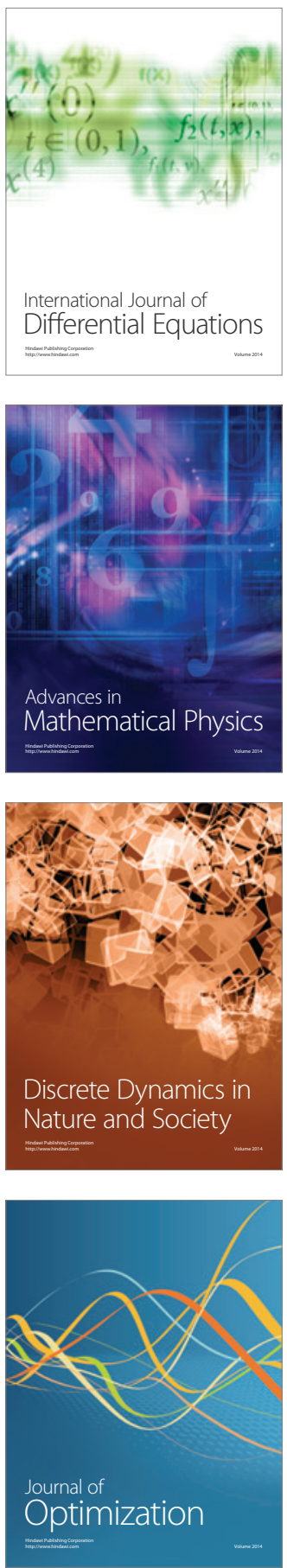